\title{
Relationship between person with dementia and adolescents: A preliminary survey of the Association Between Children and Dementia Elders (ABCDE) Project
}

Shigeki Hirano ${ }^{1,2 *}$, Keisuke Shimizu ${ }^{1}$, Noriko Murayama ${ }^{1}$, Moeno Ishikawa ${ }^{1}$, Shogo Furukawa ${ }^{2}$, Kazuho Kojima ${ }^{2}$, Ai Ishikawa ${ }^{2}$, Hitoshi Shimada $^{2,3}$, Hitoshi Shinotoh ${ }^{4}$ Koichi Kashiwado ${ }^{2,5}$, Tsuyoshi Sasaki ${ }^{6,7}$, Masaomi Iyo ${ }^{1,7}$ and Satoshi Kuwabara ${ }^{1,2}$

${ }^{1}$ Chiba Dementia Medical Centre, Chiba University Hospital, Chiba, Japan

${ }^{2}$ Department of Neurology, Graduate School of Medicine, Chiba University, Chiba, Japan

${ }^{3}$ Department of Functional Brain Imaging Research, Clinical Research Cluster, National Institute of Radiological

${ }^{4}$ Sciences, National Institutes for Quantum and Radiological Science and Technology, Chiba, Japan

${ }^{5}$ Neurology Clinic Chiba, Chiba, Japan

${ }^{6}$ Kashiwado hospital, Chiba, Japan

${ }^{7}$ Department of Child Psychiatry, Chiba University Hospital, Chiba, Japan

${ }^{8}$ Department of Psychiatry, Graduate School of Medicine, Chiba University, Chiba, Japan

\begin{abstract}
Objectives: The aim of this study was to clarify the relationship and psychological reaction of person with dementia and their grandchildren by administering a questionnaire to the primary caregiver.

Methods: A total of 109 caregivers of people with dementia in outpatient enrolled in this study. Questionnaire collected information on age, gender, cognitive severity (Mini-Mental State Examination), clinical diagnosis, relationship of the caregiver, frequency of meeting with grandchildren, and reaction of person with dementia to spending time with their grandchildren and/or great-grandchildren, and vice versa.

Results: Eighty-four people had at least one grandchild (77.1\%) and a quarter lived with their grandchild and/or great-grandchild. Age of grandchildren increased with increasing age of person with dementia; frequency of seeing each other decreased with increasing age. While most people with dementia were reported to look happy when spending time with their grandchildren (94.5\%), young people tended to be more non-reactive $(26.0 \%)$. Non-reactive grandchildren were more prevalent in the older dementia age group ( $\mathrm{p}<0.001)$. Nevertheless, the majority of grandchildren $(74.0 \%)$ enjoyed spending time together.
\end{abstract}

Conclusions: People with dementia enjoy spending time with their grandchildren, although older grandchildren are less reactive in these situations.

\section{Introduction}

The old age population is growing rapidly in developed countries and the proportion of the number of elders among whole population in Japan has become the highest in the world since 2000 [1,2]. In Japan, the proportion of the population aged over 65 years was $26 \%$ in 2015 while the developed western countries range between $13 \%$ to $20 \%$, and this figure is estimated to raise in Japan up to $38.8 \%$ by 2050 [2]. Dementia presents as a slow, progressive cognitive impairment that affects daily living, one of the most hazardous risk factors being ageing. Hence, number of individuals with dementia is growing rapidly. However, the local demand for social services is high in respect to actual service supply. Therefore, an even greater amount of social care will be necessary in global but especially in Japan as the population ages in the near future.

Behavioural and psychological symptoms of dementia (BPSD) are defined as a group of symptoms of disturbed perception, thought content, mood, or behaviour. The presence of BPSD in people with dementia may result in distress in their caregivers, as well as in the person themselves. BPSD may cause profound care burden and may give rise to institutionalization [3-5]. The symptoms of BPSD exacerbate when caregivers treat people with dementia inappropriately. Conversely, people with dementia may ameliorate their abnormal behaviours when in a supportive environment and caregiver characteristics also influence dementia behaviours. Recent evidence shows that non-pharmacological interventions, including simple psychological interventions, staff-training programs, reminiscence, personalized leisure activities, person-centered care training, and social interaction have become first-line practical management strategies against BPSD

${ }^{\star}$ Correspondence to: Shigeki Hirano, 1-8-1 Inohana, Chuo-ku, Chiba-shi, Chiba, Japan, Tel: +81-43-226-2736; Fax: +81-43-226-2738, E-mail: s_hirano@chiba-u.jp

Key words: dementia, children, relationships, questionnaire

Received: March 18, 2020; Accepted: March 25, 2020; Published: April 01, 2020 
[6,7]. According to one study, caregivers whose struggling to accept their caregiving situations and dementia related problems (nonadapters) increased the hyperactive symptoms of people with dementia [8]. Moreover, caregivers with non-adapting strategies reported a lower sense of competence, a high prevalence of depressive symptoms, and higher levels of neuroticism [8].

Elders generally favor spending time with their grandchildren or small children and may remain calm and peaceful in their presence. A meta-analysis demonstrated that positive psychological interventions, such as writing gratitude letters, practicing optimistic thinking, replaying positive experiences, and socializing, may effectively boost well-being, and these benefits increase with age [9]. In addition, people with dementia may be capable of playing with young children, which may provide them with a sense of purpose [10]. However, adolescents do not usually have enough time or the opportunity to learn about dementia and when confronted with aberrant behaviours, they may feel embarrassed, anxious, or even depressed. Since adolescents often do not know how to communicate with people with dementia, the opportunities to visit with their grandparents with dementia may reduce.

In 2013, we proposed the Association Between Children and Dementia Elders (ABCDE) project with the aim of helping adolescents to understand dementia. One of the main goals of this project is to educate young children and adolescents regarding how to properly communicate with elders with dementia. In addition, a previous report showed that through caring for their grandparents with dementia, adolescents learned about attitudes toward life, underwent personal changes, reflected on ageing, learned about the importance of family, and gained knowledge about the disease [11]. Therefore, increasing the opportunity to spend time with children and adolescents may provide pleasant interactions for people with dementia, probably leading to the stabilization of aggressive or depressive symptoms and perhaps improves social well-being. Moreover, understanding dementia may improve the anxiety and depression experienced by young people and may improve non-verbal communication towards people with dementia.
The aim of this study was to investigate demographic information and clarify the frequency of visits and the reactions of grandchildren and people with dementia when spending time together. Primary caregiver (hereby referred to as "respondent") of person with dementia completed a questionnaire at an outpatient clinic visit. Briefly, the questionnaire investigated the status of the person with dementia, the frequency of visits between person with dementia and grandchildren, and how the two reacted to time spent together. The results of this survey will provide the epidemiological basis for the $\mathrm{ABCDE}$ project.

\section{Methods \\ Participants}

Study participants consisted of 110 primary caregivers who knew how the person with dementia spent their time at home. The primary physician collected demographic data. Individuals with dementia visiting the outpatient clinic of the Centre for Dementia, Chiba University Hospital $(\mathrm{n}=48)$ and five related clinics including the Neurology Clinic Chiba $(n=45)$, Kashiwado Hospital $(n=9)$, Yatsu Hoken Hospital $(n=5)$, and Chiba Tokushukai Hospital $(n=3)$. One respondent did not fill in more than two-thirds of the questionnaire and was excluded from subsequent analysis, resulting in a final sample of 109. Respondents consisted of 21 husbands, 27 wives, 18 sons, 35 daughters, 8 others, and no grandchildren. The questionnaire was administered between November 2014 and January 2015.

Chiba Prefecture is $5,157.65 \mathrm{~km}^{2}$, has 6.6 million residents, and is located east of Tokyo. The western region of Chiba is suburban and the southern part is rural. Chiba city is $271.7 \mathrm{~km}^{2}$, located between the suburban and rural districts, centre of Chiba Prefecture. Chiba city is a government ordinance-designated city with a population of approximately 1 million, in which Chiba University Hospital is located (Figure 1).

The study was approved by the Institutional Review Board of Chiba University, School of Medicine. The study method and purpose were explained in oral and written form to caregivers at the clinic and only the anonymous questionnaire was submitted.

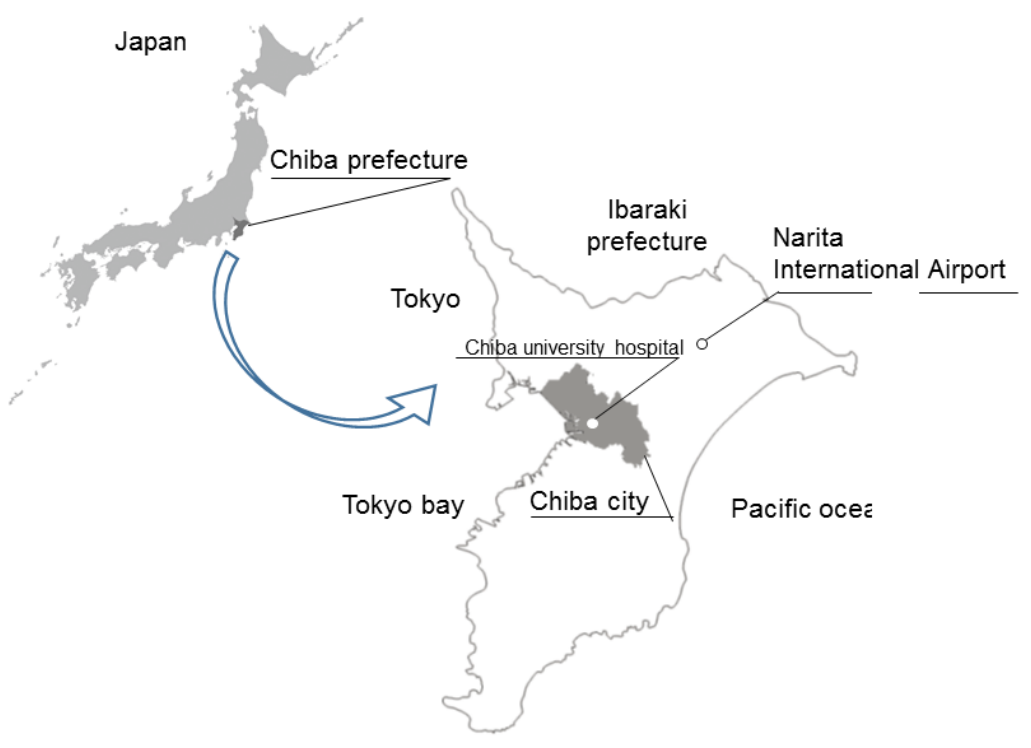

Figure 1. Map of Chiba Prefecture. Chiba prefecture is a suburban area, east of capital Tokyo. Chiba city is located in the centre of the Prefecture, in between the suburban (western) and rural (southern) regions 
Hirano S (2020) Relationship between person with dementia and adolescents: A preliminary survey of the Association Between Children and Dementia Elders (ABCDE) Project

\section{Questionnaire}

The questionnaire took 5 to 10 minutes to complete. Questionnaire items (Q1-10) were as follows: Q1. demographic information of person with dementia (age, gender, living district, clinical diagnosis, duration of illness, most recent Mini-Mental State Examination (MMSE) score [12] to measure general cognitive status, if applicable) was recorded by a physician; Q2. relationship of the respondent to the person with dementia; Q3. who lived with the person with dementia; Q4. how many children the person with dementia had; Q5. how many grandchildren the person with dementia had (respondents were asked to indicate the gender, age, and place of residence of each grandchild); Q6. how often the person with dementia met with their grandchild(ren) (multiple choice question) $[1=$ Less than once per year; $2=$ Once per year; $3=$ $2-5$ times per year; $4=6-11$ times per year; $5=$ once per month; $6=$ 2-3 times per month; $7=$ Once per week; $8=2-6$ times per week; $9=$ everyday]; Q7. how the person with dementia and their grandchild(ren) and/or great-grandchild(ren) spent time together (open-ended question); Q8. whether the person with dementia looked happy when they spent time with their grandchild(ren) (5-point Likert scale; $1=$ very happy, 2 = happy, $3=$ neither happy nor unhappy, $4=$ unhappy, 5 = very unhappy); Q9. whether the grandchild enjoyed spending time with their grandparent (5-point Likert scale; $1=$ enjoys very much, $2=$ enjoys, $3=$ neither enjoys nor is bored, $4=$ is bored, $5=$ is very bored); Q10. whether the person with dementia wanted the grandchildren to visit more frequently (5-point Likert scale; $1=$ strongly agree, $2=$ agree, 3 = equivocal, 4 = disagree, 5 = strongly disagree).

\section{Statistical analyses}

Frequencies and percentages are reported for demographic data reported in Q1, Q4-6, and Q8-10. In order to understand the influencing factors, Q8, Q9, Q10 were subsequently divided into subgroups by (1) age; (2) gender; (3) total MMSE score; and (4) whether or not the grandchild(ren) and/or great-grandchild(ren) lived with the person with dementia; these groups were then compared. People with dementia were divided into three age groups: $\leq 74,75-84$, and $\geq 85$ years. Total MMSE score was divided into four categories: $\leq 9$, $10-19,20-23$, and $\geq 24$. Statistical analyses consisted of Mann-Whitney $\mathrm{U}$-test for gender and living status and Kruskal-Wallis test with posthoc Bonferroni correction. To further investigate the difference in reaction of the person with dementia vs. their grandchildren and/or great-grandchildren to spending time together, the Wilcoxon signedrank test was applied. P-values of $<0.05$ were considered statistically significant. All statistical tests were performed using IBM SPSS version 24 for Windows (IBM Corp., Armonk, NY, USA).

\section{Results}

\section{Demographic data of people with dementia}

People with dementia $(n=107)$ had a mean age of 77.9 years (SD 6.8) and consisted of 33 males and 74 females; demographic data were missing for two people. Median duration of illness was 3.0 years (range: $0.25-18.0, \mathrm{n}=98$ ); disease duration frequencies were as follows: $<1$ year: $7 \%, 1-3$ years: $38 \%, 3-5$ years: $26 \%, \geq 5$ years: $21 \%$, unknown: $8 \%$. Clinical diagnoses were as follows, 70 with Alzheimer's disease (64.2\%), 11 dementia with Lewy bodies (10.1\%), nine with mild cognitive impairment (8.3\%), five with mixed-type dementia (Alzheimer's disease and vascular dementia) (4.6\%), four with corticobasal syndrome (3.7\%), nine with undetermined diagnosis $(8.3 \%)$, and one with semantic dementia. Median MMSE score was 21.0 (range: 0 - 30, $\mathrm{n}=98$ ); MMSE scores were as follows: $\geq 24$ : 32 (32.7\%), 23-20: 31 (31.6\%), 10-19: 27 $(27.6 \%), \leq 9: 8(8.2 \%), 11$ data were missing.

\section{Demographic data of grandchildren}

Most people with dementia had at least one child (105 out of $107 ; 98.1 \%)$. Eighty-four people had grandchildren, totalling 279 grandchildren. Numbers of grandchildren; 25 people had no grandchildren (22.9\%), 9 had one grandchild (8.3\%), 26 had 2 grandchildren (23.9\%), 16 had $3(14.7 \%), 16$ had $4(14.7 \%), 17$ had $\geq 5$ grandchildren (15.6\%). Twenty-one out of the 84 people $(25.0 \%)$ with grandchildren lived with their grandchildren. Age were higher in people with dementia who lived with their grandchild(ren) (median age: 83) compared to those who lived separately (median age: 78) ( $\mathrm{p}$ $<0.001$, Mann-Whitney U test), whereas MMSE score did not differ between those two subgroups ( $\mathrm{p}=0.68$, Mann-Whitney $\mathrm{U}$ test).

Ages of grandchildren were as follows: $\leq 8$ years: $20.8 \%$, 9-12 years: $12.2 \%, 13-15$ years: $7.5 \%, 16-18$ years: $9.3 \%, \geq 19$ years: $50.2 \%$. Ages of grandchildren positively correlated with the people with dementia $\left(\mathrm{R}_{\mathrm{s}}=\right.$ $0.386, p<0.001$, Spearmann's rank correlation test). Figure $2 \mathrm{~A}$ shows the ages of grandchildren in relation to their grandparent with dementia. Of note, the percentages of grandchildren above the age of 19 years were $19.6 \%, 50.6 \%$, and $72.1 \%$ in people aged $\leq 74,75-84$, and $\geq 85$ years, respectively. The percentages of grandchildren below the age of 8 years were $41.3 \%, 18.6 \%$, and $9.8 \%$ in grandparents with dementia aged $\leq 74,75-84$, and $\geq 85$ years, respectively. Figure $2 \mathrm{~B}$ shows the frequency by which person with dementia saw their grandchild(ren). In all age groups, the most predominant answer was $2-5$ times annually, reported in $33.3 \%, 41.3 \%$, and $40.0 \%$ of people aged $\leq 74,75-84$, and $\geq 85$ years, respectively.

(A)

(B)
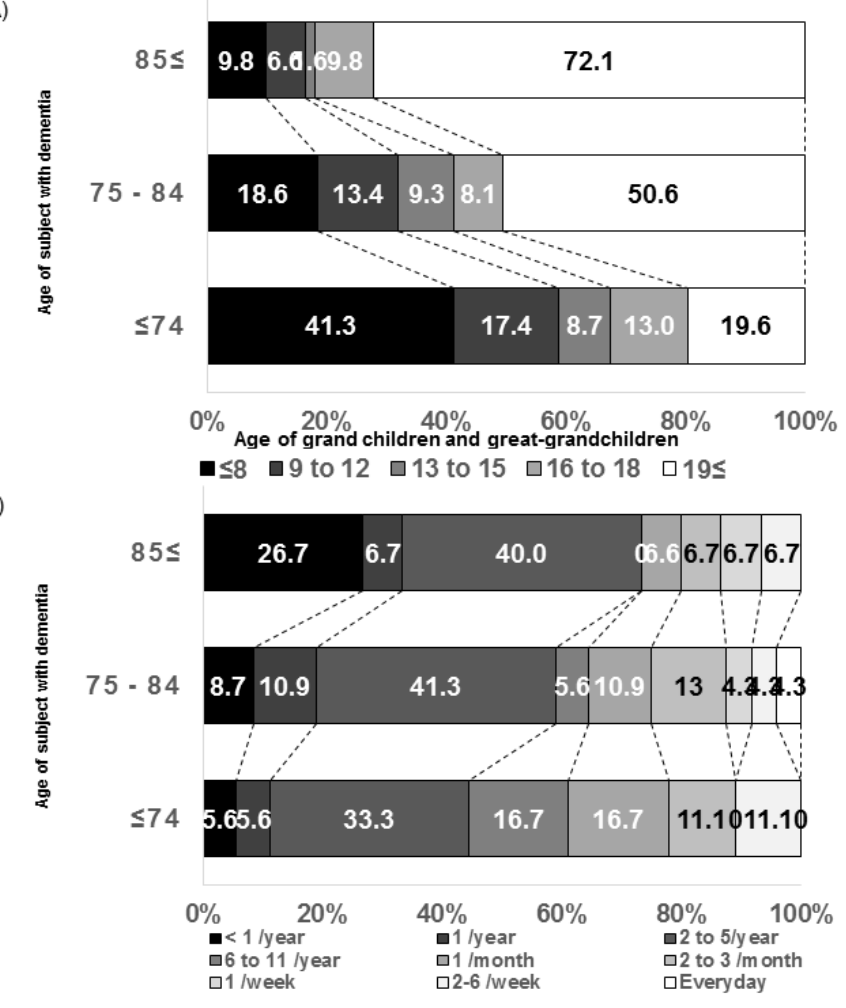

Figure 2.

(A) Relationship between age of the person with dementia and the age of grandchildren and great-grandchild(ren)

(B) Relationship between age of the person with dementia and the frequency of the grandchildren visiting them 
Figure 3 shows numbers (all 119 answers including multiple answers per respondent) of the ways in which grandchild(ren) and/ or great-grandchild(ren) spent time with person with dementia (i.e., Q7). Dining at home was the most frequent answer $(\mathrm{n}=$ $29)$, followed by making conversation $(n=19)$ and shopping ( $=14)$. Some of the answers, such as watching television $(\mathrm{n}=8)$, observation $(n=4)$, receive money $(n=2)$, were passive activities. Reactions of person with dementia spending time with their grandchild(ren) and/or great-grand child(ren) (i.e., Q8), 69 out of 73 answers (94.5\%) indicated that person with dementia looked very happy' or 'happy.' None reported to look 'unhappy' and the remaining people $(n=4)$ were therefore reported to look 'neither happy nor unhappy'. Age ( $\mathrm{p}=0.413, \mathrm{n}=71)$, gender $(\mathrm{p}=0.199, \mathrm{n}=73)$, severity of cognitive impairment $(p=0.078, n=67)$ of the person with dementia, and whether or not they lived with their grandchildren $(\mathrm{p}=0.472, \mathrm{n}=$ 72) did not differ across reported responses to this question.

In terms of adolescents' reactions to spending time with their grandparents with dementia, Q9 showed that 54 out of 73 answers given by respondents were either 'enjoys very much' or 'enjoys' (74.0\%); 19 answered 'neither enjoys nor is bored', (26.0\%). None of the adolescents were reported to be bored. Responses to this question differed significantly between people with dementia aged $\leq 74$ years and $\geq 85$ years $(\mathrm{p}<0.05, \mathrm{n}=71)$; more adolescents in the younger age group reported to enjoy the time spent with person with dementia in the younger age group. Gender $(p=0.543, n=73)$, severity of cognitive impairment $(\mathrm{p}=0.507, \mathrm{n}=67)$ of people with dementia, and whether they lived with their grandchildren $(\mathrm{p}=0.639, \mathrm{n}=72)$, did not differ across reported responses to this question. Respondents reported that the people with dementia enjoyed spending time with the adolescents more than the adolescents did $(\mathrm{p}<0.001, \mathrm{n}=73)$.

Finally, responses regarding whether the person with dementia wanted their grandchildren to visit more frequently (i.e., Q10) are shown in Figure 4 . The majority $(81.9 \%)$ of respondents answered either 'strongly agree' or 'agree.' Age $(\mathrm{p}=0.364, \mathrm{n}=70)$, gender $(\mathrm{p}=916$, $\mathrm{n}=72)$, severity of cognitive impairment $(\mathrm{p}=0.143, \mathrm{n}=66)$ of person

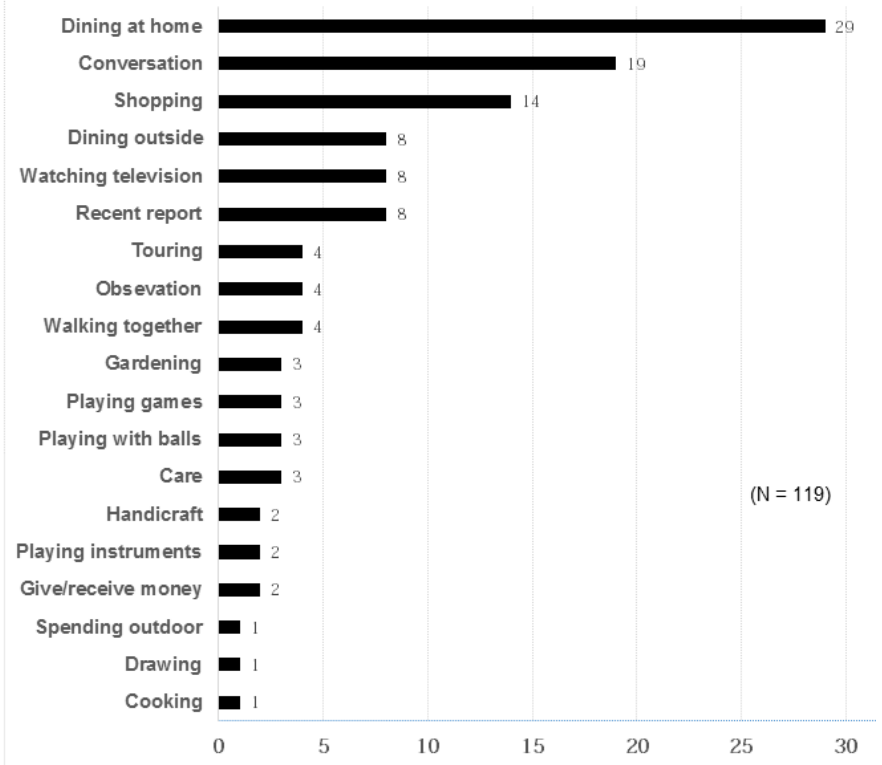

Figure 3. How the child spent time with their grandparent or great-grandparent with dementia

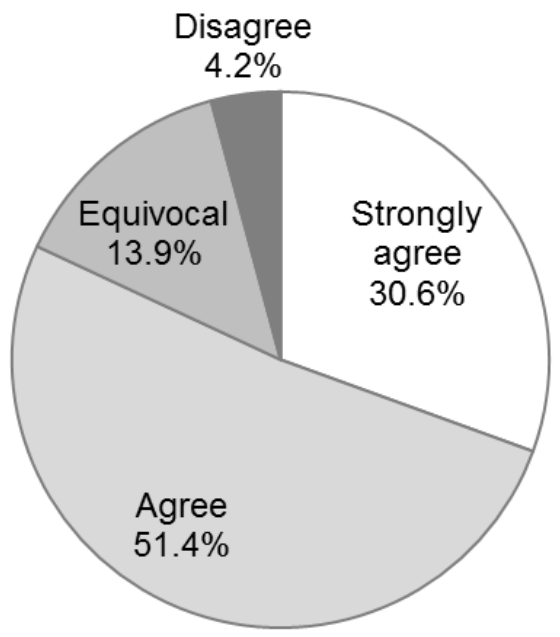

Figure 4. Responses to whether caregiver wants the grandchildren to visit more frequently $(\mathrm{n}=72)$

with dementia, and whether they lived with their grandchildren $(\mathrm{p}=$ $0.185, \mathrm{n}=72$ ) did not differ across reported responses to this question.

\section{Discussion}

The present results indicate that people with dementia express happiness when they are able to see their grandchild(ren) and/or greatgrandchild(ren), irrespective of their age, gender, severity of dementia, or whether or not they live with their grandchild(ren). The vast majority of people with dementia were reported to look either 'very happy' or 'happy' when spending time with their grandchild(ren). This contrasted with the results for grandchild(ren) and great-grandchild(ren), in which more than a quarter were reported to show no emotional response. In other words, despite adolescents not showing much emotional response to spending time together, people with dementia seemed happy nevertheless. Grandparents, in general, tend to have a good relationship with their grandchildren [13]. People with dementia may play role as a carer when interacting with adolescents. Further, it may give people with dementia the opportunity to teach their grandchildren lessons such as morality, simple manners, and how to play with things they played with when they were young.

Older people with dementia has grandchildren of higher age, shown in this study. As adolescent get older, they tend to become overwhelmed into their work and their own lives which makes it difficult to frequently see their (great-)grand parents, also shown in this study. The frequency by which relatives meet is related not only to the distance between their respective residences, but also to their familiarity with each other [14]. Since children are usually unfamiliar with the knowledge about dementia, they may be withdrawn or anxious to see the person with dementia. Therefore, in order to reinforce the interactions between adolescents and elders with dementia, it may be favorable for adolescents to understand the background or reason behind the behaviour of people with dementia. In previous research, administering appropriate non-pharmacological interventions to caregivers reduced agitation and increased pleasure of people with dementia $[15,16]$. The present study demonstrated that responses to spending time together were more positive in younger people with dementia. Since younger people with dementia tend to have younger grandchildren, this finding suggests the importance of early education of children regarding dementia which may help maintaining the relationship. This education could also allow 
young children without having any relatives with dementia to detect the future appearance of dementia symptoms in their relatives.

Education regarding the reasons for symptoms of dementia and how to react to the behaviour of people with dementia is valuable for the caregiver, needless to say that this notion applies to the adolescents. However, because most of the people with dementia visits hospital without small children, children scarcely have opportunity to learn about dementia from dementia experts. Additionally, in adolescents, experience caring for grandparents with dementia creates closer family bonds, greater empathy for older adults, significant motheradolescent bonding, and peer relationship selection and maintenance [17]. Educating adolescents would also indirectly teach their parents, usually the primary caregivers for the person with dementia who may not have enough time to learn about dementia. Moreover, it would be a good opportunity for the adolescents to better understand disability, age-related health issues, and to be more empathic towards people with disability. As people with dementia age, their grandchildren grow up and may become too busy to visit and/or see each other [18]. When people become busy, it is difficult to find the time to learn about dementia. Isolation of the caregiver from the entire family system is also an important issue to prevent. We believe that education of adolescents regarding dementia may relieve adolescents and make them feel more comfortable when confronted with the aberrant behaviour of people with dementia. In turn, this would increase the opportunity to visit and efficiently communicate with their grandparents, thereby reducing the isolated feeling of caregiver. Nevertheless, it is also of note for adults to keep small children safe while visiting people with dementia.

Limitations of this study include the lack of an age-matched control group. Adolescents' loss of interest may be irrelevant to their grandparents' dementia, and is solely due to the natural course of development. In order to clarify this issue, future studies should either provide an age-matched control group or conduct a prospective, longitudinal dementia education study to improve awareness of dementia among adolescents. Sustaining better relationships between older and younger generations is beneficial to the wellbeing of society. Another limitation is that the questionnaire was conducted in an outpatient setting, which makes the respondents more likely to be a family caregiver. Elders with dementia who live alone or who have no relatives may have other issues, such as different medical intervention and health care situations $[19,20]$, which should be addressed in future research. Since most respondents were relatives, answers maybe biased to preferable one, although assessments in dementia researches tend to be rated by caregivers, such as in the clinical dementia rating and in the neuropsychiatric inventory. This question shall be left for the future research dealing with institutionalized people with dementia whose respondents are not relatives. Additionally, one could argue that people with dementia might be happy whether or not grandchild(ren) were present or not and may felt happy from solely seeing the caregiver. Whether meeting adolescent and people with dementia may actually improve negative feelings of adolescents and improves behavioural symptoms of dementia is unknown and shall be solved in the future studies. Future perspectives of this study are to evaluate grandchildren and to evaluate whether education modifies the psychological states of children. Establishment of education program for kids are needed. Prospective educational study in the grandchildren of people with dementia and directly measuring BPSD status of the people with dementia shall elucidate many aspects of this project.

In conclusion, people with dementia feel happy when they spend time with grandchild(ren) and/or great-grandchild(ren). This finding was in contrast with the emotionless response observed in over a quarter of young people. This tendency became more prominent when grandchild(ren) got older.

\section{Acknowledgement}

The present work was supported in part by Kashiwado medical research scholarship, Kashiwado Memorial Foundation and by Community Oriented Education and Research Fund, Centre of Community (COC) Program, Chiba University. Authors would like to thank Mrs. Kaoru Tomita, Chief of Integrated Community Care Division, Chiba city, for her continuous advice and support.

\section{References}

1. Balas MC, Burke WJ, Gannon D, Cohen MZ, Colburn L, et al. (2013) Implementing the awakening and breathing coordination, delirium monitoring/management, and early exercise/mobility bundle into everyday care: opportunities, challenges, and lessons learned for implementing the ICU Pain, Agitation, and Delirium Guidelines. Crit Care Med 41: S116-127. [Crossref]

2. Cabinet Office GOJ (2015) Annual Report on the Ageing Society.

3. Buhr GT, Kuchibhatla M, Clipp EC (2006) Caregivers' reasons for nursing home placement: clues for improving discussions with families prior to the transition. Gerontologist 46: 52-61. [Crossref]

4. Rabins PV, Mace NL, Lucas MJ (1982) The impact of dementia on the family. JAMA 248: 333-335.

5. Steele C, Rovner B, Chase GA, Folstein M (1990) Psychiatric symptoms and nursing home placement of patients with Alzheimer's disease. Am J Psych 147: 1049-1051. [Crossref]

6. Ballard C, Corbett A (2013) Agitation and aggression in people with Alzheimer's disease. Curr Opin Psychiatry 26: 252-259. [Crossref]

7. Testad I, Corbett A, Aarsland D, Lexow KO, Fossey J, et al. (2014) The value of personalized psychosocial interventions to address behavioural and psychological symptoms in people with dementia living in care home settings: a systematic review. Int Psychogeriatr 26: 1083-1098. [Crossref]

8. de Vugt ME (2004) Do caregiver management strategies influence patient behaviour in dementia? Int J Geriatr Psychiatry 19: 85-92. [Crossref]

9. Sin NL, Lyubomirsky S (2009) Enhancing well-being and alleviating depressive symptoms with positive psychology interventions: a practice-friendly meta-analysis. $J$ Clin Psychol 65: 467-487. [Crossref]

10. Celdran M, Villar F, Triado C (2014) Thinking about my grandparent: How dementia influences adolescent grandchildren's perceptions of their grandparents. J Ageing Stud 29:1-8 [Crossref]

11. Celdran M, Triado C, Villar F (2009) Learning from the disease: lessons drawn from adolescents having a grandparent suffering dementia. Int J Aging Hum Dev 68: 243 259. [Crossref]

12. Folstein MF, Folstein SE, McHugh PR (1975) "Mini-mental state". A practical method for grading the cognitive state of patients for the clinician. J Psyc Res 12: 189-198. [Crossref]

13. Creasey GL, Koblewski PJ (1991) Adolescent grandchildren's relationships with maternal and paternal grandmothers and grandfathers. Journal of adolescence 14: 373 387. [Crossref]

14. Argyle M, Henderson M (1985) The Anatomy of Relationships: And the Rules and Skills Needed to Manage Them Successfully. William Heinemmann, London.

15. Cohen-Mansfield J, Thein K, Marx MS, Dakheel-Ali M, Freedman L (2012) Efficacy of nonpharmacologic interventions for agitation in advanced dementia: a randomized, placebo-controlled trial. J Clin Psychiatry 73: 1255-1261. [Crossref]

16. Gitlin LN, Winter L, Burke J, Chernett N, Dennis MP, et al. (2008) Tailored activities to manage neuropsychiatric behaviours in persons with dementia and reduce caregiver burden: a randomized pilot study. Am J Geriatr Psychiatry 16: 229-239. [Crossref]

17. Beach DL (1997) Family caregiving: the positive impact on adolescent relationships. Gerontologist 37: 233-238. [Crossref]

18. Peterson K, Hahn H, Lee AJ, Madison CA, Atri A (2016) In the Information Age, do dementia caregivers get the information they need? Semi-structured interviews to determine informal caregivers' education needs, barriers, and preferences. $B M C$ geriatrics 16: 164 
Hirano S (2020) Relationship between person with dementia and adolescents: A preliminary survey of the Association Between Children and Dementia Elders (ABCDE) Project

19. Cermakova P (2017) Living Alone with Alzheimer's Disease: Data from SveDem, the Swedish Dementia Registry Journal of Alzheimer's disease: JAD 58:1265-1272. [Crossref]
20. Eichler T (2016) Living Alone with Dementia: Prevalence, Correlates and the Utilization of Health and Nursing Care Services. J Alz Dis 52: 619-629. [Crossref]

Copyright: $\bigcirc 2020$ Hirano S. This is an open-access article distributed under the terms of the Creative Commons Attribution License, which permits unrestricted use, distribution, and reproduction in any medium, provided the original author and source are credited. 